Genes \& Nutrition Vol. 1, No.3/4, pp. 141-142, 2006

ISSN 1555-8932 print, Copyright (C) 2006 by New Century Health Publishers, LLC www.newcenturyhealthpublishers.com

All rights of reproduction in any form reserved

\title{
Literature Highlights
}

\section{ASIDEWAYS GLANCE \\ Yula Sambuy \\ sambuy@inran.it}

This issue of Sideways Glance on the role of Perilipin in adipose lipid metabolism coincides with the recent largely deserved award from the United States Department of Agriculture (USDA) to Dr. Jose Ordovas of the Jean Mayer USDA Human Nutrition Research Center on Aging at Tufts University in Boston, Mass. for his many contributions to nutrigenomics. The USDA Secretary's Honor Award recognizes the fundamental contributions that Dr Ordovas has made to the understanding of nutrient gene interactions in humans. Two recent papers from Dr Ordovas' group are discussed below reporting the association between genetic variations in adipocyte perilipin and the response to dietary interventions on the susceptibility to developing insulin resistance or obesity in selected population groups.

\section{PERILIPIN, A CENTRAL AND MULTIFACETED PROTEIN IN ADIPOSE LIPID METABOLISM}

A great deal of attention has recently been placed on the search for associations between genetic variants, represented by single nucleotide polymorphisms (SNPs), and major pathological conditions in response to particular dietary conditions. In this rapidly expanding field, it is becoming clear that the potential benefit of this type of knowledge to future public health intervention and to clinical practice lies not only in the numbers but in the search for multiple interactions and in the need to substantiate epidemiological nutrigenomic findings with physiological and biochemical approaches aimed at identifying the mechanisms underlying such interactions at the level of the cell and the organism. Regulation of adipocyte lipolysis is central in whole body energy homeostasis and it is vital to metabolic health since fatty acids released during adipocyte lipolysis also function as modulators of glucose and insulin action, as well as in insulin production. The disregulated release of fatty acids from adipocytes that occurs in obesity is implicated in the etiology of obesity-related complications, including type 2 diabetes.

Perilipin (PLIN) is the predominant protein associated with adipocyte lipid droplets. Two alternatively spliced variants, PLIN $A$ and PLIN B, are expressed in adipocytes the former being the most abundant form. In the absence of lipolytic stimulation PLIN
A inhibits lipolysis by acting as a barrier against hydrolysis of the stored triacylglycerides by lipases. Catecholamines stimulate lipolysis by binding to beta-adrenergic receptors in adipocytes resulting in upregulation of adenyl cyclase and activation of cAMP-dependent phosphokinase A (PKA). PKA activity is thought to increase lipolysis simultaneously by phosphorylating PLIN A and the hormone sensitive lipase (HSL). Upon PKA activation, HSL is translocated to the lipid droplets where the interaction with phosphorylated PLIN A allows the lipolytic action to proceed. Thus, PLIN functions both as repressor of basal lipolysis and as enhancer of PKA-stimulated lipolysis

The importance of PLIN in adipose lipid metabolism had emerged from the first studies with PLIN knockout mice. The adipose mass of PLIN null mice was reduced and stimulated lipolysis was attenuated, while they exhibited an increased tendency to develop glucose intolerance and peripheral insulin resistance upon feeding a high fat diet (Tansey et al., 2001). Efforts have therefore been directed at finding associations between PLIN SNPs and predisposing conditions to pathologies characterized by unbalanced lipid metabolism.

While direct associations between PLIN genotype and insulinresistance or obesity have not clearly emerged, an association between diet response and perilipin polymorphism has been observed in a recent study. Asian women with PLIN 11482G$>\mathrm{A} / 14995 \mathrm{~A} \longrightarrow \mathrm{T}$ polymorphisms had higher susceptibility to insulin resistance in association with a diet with high short chain fatty acids (SFA) to carbohydrate ratio (Corella et al., 2006). Similarly, the same group had previously reported that the minor A-allele at the PLIN locus $(11482 \mathrm{G}>\mathrm{A})$ was associated with lower baseline body weight (BW) and with resistance to weight loss, suggesting that this polymorphism may predict outcome of BW reduction strategies based on low-energy diets.(Corella et al., 2005).

A particular response to dietary intervention in association with specific genotypes may warrant further studies aimed at the identification of the underlying mechanisms. Although the $11482 \mathrm{G}>\mathrm{A}$ PLIN polymorphism is located in an intron, obese subjects that were homozygous for the A allele were found to 
have reduced PLIN protein expression and higher rates of hormone-stimulated lipolysis in adipocytes (Mottagui-Tabar et al., 2003). Unfortunately, studies aimed at identifying molecular mechanisms of perilipin regulation in lipolysis need to be performed in adequate tissue-specific cell models, and these are not easy to perform in adipose tissues from individuals with different PLIN polymorphisms.

An elegant study recently published has clarified some important points of regulation of PLIN A action. Employing adenoviral and transgenic expression of a PLIN construct deficient in all six serines that are phosphorylated by PKA in stable murine embryonic fibroblastic differentiated into adipocytes obtained from PLIN knockout mice, it was demonstrated that PKAdependent phosphorylation is not required for HSL translocation to the lipid droplets (LD), but is essential for lipolytic action(s) of HSL associated with the LD (Miyoshi et al., 2006). In addition, cross-linking and immunoprecipitation studies suggested that the PKA-dependent phosphorylation of PLIN may induce conformational changes and alter its spatial relationship with HSL at the LD surface, promoting access of the lipase to the stored neutral lipids, thereby initiating lipolysis.

The independence of HSL translocation from PLIN A phosphorylation was also indirectly confirmed by the effects of the phosphatase (PPAse) inhibitor calyculin on primary and differentiated rat adipocytes. PPAse inhibition resulted in hyperphosphorylation of PLIN A but did not cause HSL translocation, while it stimulated lipolysis probably by the HSL already associated with the lipid droplets that accounts to about $40 \%$ of total HSL (He et al., 2006). This work has also highlighted the existence of a fine balance between PPases (themselves under hormonal control) and kinases in the regulation of adipocyte lipolysis. As an example, the anti-lipolytic effect of insulin is mediated via dephosphorylation of both HSL and PLIN (references in Aboulaich et al., 2006).

A Swedish group has recently identified a different anti-lipolytic effect of insulin that involves the PLIN B isoform at the plasma membrane (Aboulaich et al., 2006). PLIN B is the minor splice variant in adipocytes but it is highly enriched at the plasma membrane. Proteomic and mass spectrometric analysis of phosphorylated peptides from plasma membrane proteins was performed in insulin stimulated primary human adipocytes obtained from subcutaneous abdominal fat tissue. Phosphorylation of PLIN B on a cluster of three threonine residues was stimulated by insulin and led to translocation of PLIN B to the plasma membrane, although the kinase responsible was not investigated. (Aboulaich et al., 2006). Phosphorylated PLIN B at the plasma membrane is localized in caveolae in which added long chain fatty acids are rapidly converted to triglycerides, thus its translocation in response to insulin may protect the freshly synthesized triglycerides from hydrolysis by HSL found to be associated to triglyceride-synthesizing caveolae (Ortegren et al., 2006).

Using different experimental approaches, all papers discussed above have shown the central role of PLIN in the control of lipolysis under different physiological stimuli. Thus changes in the diet are likely to affect this complex regulatory network and may shift the delicate balance between health and pathological conditions. In this context, it is particularly interesting the emerging role of the genetic background in the PLIN genes polymorphisms in influencing the physiological response to dietary interventions.

\section{REFERENCES}

Aboulaich, N., Vener, A. V., and Stralfors, P. (2006). Hormonal control of reversible translocation of perilipin B to the plasma membrane in primary human adipocytes. Journal of Biological Chemistry 281, 11446-11449.

Corella, D., Qi, L., Sorli, J. V., Godoy, D., Portoles, O., Coltell, O., Greenberg, A. S., and Ordovas, J. M. (2005). Obese subjects carrying the $11482 \mathrm{G}>\mathrm{A}$ polymorphism at the perilipin locus are resistant to weight loss after dietary energy restriction. Journal of Clinical Endocrinology and Metabolism 90, 5121-5126.

Corella, D., Qi, L., Tai, E. S., Deurenberg-Yap, M., Tan, C. E., Chew, S. K., and Ordovas, J. M. (2006). Perilipin gene variation determines higher susceptibility to insulin resistance in Asian women when consuming a high-saturated fat, low-carbohydrate diet. Diabetes Care 29, 1313-1319.

He, J., Jiang, H., Tansey, J. T., Tang, C., Pu, S., and Xu, G. (2006). Calyculin and okadaic acid promote perilipin phosphorylation and increase lipolysis in primary rat adipocytes. Biochimica Biophysica Acta 1761, 247-255.

Miyoshi, H., Souza, S. C., Zhang, H. H., Strissel, K. J., Christoffolete, M. A., Kovsan, J., Rudich, A., Kraemer, F. B., Bianco, A. C., Obin, M. S., and Greenberg, A. S. (2006). Perilipin promotes hormone-sensitive lipase-mediated adipocyte lipolysis via phosphorylation-dependent and -independent mechanisms. Journal of Biological Chemistry 281, 15837-44.

Mottagui-Tabar, S., Ryden, M., Lofgren, P., Faulds, G., Hoffstedt, J., Brookes, A. J., Andersson, I., and Arner, P. (2003). Evidence for an important role of perilipin in the regulation of human adipocyte lipolysis. Diabetologia 46, 789-797.

Ortegren, U., Yin, L., Ost, A., Karlsson, H., Nystrom, F. H., and Stralfors, P. (2006). Separation and characterization of caveolae subclasses in the plasma membrane of primary adipocytes; segregation of specific proteins and functions. FEBS Journal 273, 3381-3392.

Tansey, J. T., Sztalryd, C., Gruia-Gray, J., Roush, D. L., Zee, J. V., Gavrilova, O., Reitman, M. L., Deng, C. X., Li, C., Kimmel, A. R., and Londos, C. (2001). Perilipin ablation results in a lean mouse with aberrant adipocyte lipolysis, enhanced leptin production, and resistance to diet-induced obesity. Proceedings National Academy Science USA 98, 6494-6499. 